\title{
Singularities of Legendre varieties, of evolvents and of fronts at an obstacle
}

\author{
V. I. ARNOL'D \\ Department of Mathematics and Mechanics, Moscow State University, \\ Lenin Hills 117234, Moscow, USSR
}

(Received 25 May 1982)

To the memory of V.M. Alexeyev

Abstract. The Legendre manifolds are the maximal integral submanifolds of a contact manifold. The shortest path problem on a manifold with boundary leads to Legendre varieties. We find normal forms of their generic singularities in terms of binary forms invariants theory.

The fronts are the hypersurfaces, equidistant from a given one. We consider fronts in a medium containing an obstacle (i.e. in a manifold with a boundary). For instance, if the obstacle is bounded by a plane curve, the fronts are its evolvents.

The Legendre manifold of a front is the set of all its tangent planes. A smooth front may acquire singularities while travelling through a smooth medium, but its Legendre manifold remains non-singular. At an obstacle, however, even the Legendre manifold may become singular. In this paper we classify generic singularities of Legendre varieties thus defined. We will describe below the normal forms for the singularities in terms of irreducible SL $(2, \boldsymbol{R})$-modules. More precisely, we define natural contact structures in the manifolds of odd degree 0 -dimensional projective hypersurfaces and in some manifolds of polynomials in one variable. We will prove that the singularities of the Legendre varieties at generic obstacles are diffeomorphic to those of the varieties of polynomials admitting high multiplicity roots.

The simplicity of the final results is rather misleading; the polynomials, and even their degrees, are hidden. Even though they are known to exist it is still difficult to find them from geometrical considerations.

The relation of the obstacle problem to $\mathrm{SL}(2, R)$-modules was discovered as a result of a series of works [1-5] featuring geometrical observations based on the resemblance of bifurcation diagrams occurring in different theories, strange cancellations of many terms in long calculations due to some properties of the varieties of polynomials with multiple roots, which seem to be new for the algebraists and new concepts in symplectic and contact geometry, namely the 'triads' of Guivental, describing families of rays and of fronts at obstacle points. 
The future general theory of the singularities for Legendre varieties seems to be an extension of the classification of the triads presented below, rather than a theory of Legendre ideals or of the generating families hierarchy (both of which are drastically different from the triad classification).

\section{The obstacle problem}

Let $\Gamma$ be a smooth hypersurface in Euclidean space $R^{n}$. Let us consider the length $S$ of the shortest path from a fixed set to a variable end point, avoiding the obstacle bounded by $\Gamma$. The study of the singularities of $S$ as a function of the end point leads to the following problem. Let us consider a family of geodesics on $\Gamma$ orthogonal to some hypersurface on $\Gamma$. The straight lines, tangent to these geodesics, define an $(n-1)$-parameter family of rays in $R^{n}$, namely the family of all normals to some front hypersurfaces in $R^{n}$. The problem is to study the singularities of these fronts. Example 1. Let the obstacle be bounded by a generic plane curve $\Gamma(n=2)$. The fronts are the evolvents of $\Gamma$. They have singularities of order $3 / 2$ at generic points of $\Gamma$ (Huyghens). A generic curve may have some inflection points. A calculation shows that the fronts have singularities of order $5 / 2$ at inflectional tangent points. Example 2. Let the obstacle be bounded by a generic surface $\Gamma$ in the 3 -space $(n=3)$. The fronts are surfaces with cuspidal edges. These edges are of order $3 / 2$ at generic points of $\Gamma$. Our one-parameter family of geodesics covers a domain in $\Gamma$. The geodesic direction may become asymptotic along some curve in this domain. The rays tangent to the geodesics at the points of this curve have asymptotic directions. The fronts' singularities at the points of an asymptotic ray are edges of order $5 / 2$ (see [1], [2]), unless the ray is 'bi-asymptotic' (this may happen at some points of our curve) in which case the singularity is more complicated. The main object of our study is the contact geometry of these complicated singularities and of their higher dimensional counterparts.

\section{The contact geometry}

We describe here some basic notions we need from contact geometry. For the proofs and for more details see [6], [7].

Definition. A contact structure on a manifold is a field of tangent hyperplanes (called contact planes), which is 'maximally non-integrable' that is, if the field of contact planes is locally defined as the zeros of an 1-form $\alpha$, then the 2-form $d \alpha$ is non-degenerate on each contact plane.

Example 1. A contact element with contact point $P$ on a manifold is a linear hyperplane in the tangent space at $P$. Let us consider the manifold of all contact elements on $V$. We denote it by $P T^{*} V$ (the projectivized cotangent bundle of $V$ ).

Let us define a field of tangent hyperplanes on $P T^{*} V$; the element velocity belongs to the field hyperplane if and only if its contact point velocity belongs to the element.

This field is a contact structure on $P T^{*} V$, called the natural contact structure of the contact elements bundle. 
Example 2. Now let us consider a hypersurface in a contact manifold, transversal to the contact planes so that the intersections of tangent planes to the hypersurface with contact ones have codimension one in both. The $d \alpha$ form defines a symplectic structure in the contact plane $\alpha=0$ which is unique up to a non-zero multiple, as is $\alpha$ itself. The $d \alpha$-skeworthocomplement to the intersection with the tangent space does not depend on this multiple; it is called the hypersurface characteristic direction.

The integral curves of the characteristic directions field are called the hypersurface characteristics. Thus a hypersurface in a contact space transversal to the contact planes is locally fibred in characteristics.

The base space of this fibration has a natural contact structure. This structure is given by the projections to the base along the characteristics of the intersections of the contact planes with tangent planes (use the Stokes formula).

Thus the characteristics space of a hypersurface in a contact manifold inherits its contact structure from the latter.

Example 3. Let us consider the pseudo-Euclidean space-time bundle of contact elements and the hypersurface formed by all light elements. The characteristics space is well defined globally in this case. One may identify it with the bundle of all transversally oriented contact elements over the physical space (the isochrone).

Indeed, a characteristic is defined by a light velocity motion. So it is defined by its point of intersection with one of the isochrones (say, $t=0$ ) and by its physical space velocity vector. The length of the latter vector is fixed, and the vector is defined by the isochrone contact element transversally oriented, orthogonal to this vector.

Proposition. The identification thus defined identifies the contact structure of the space of characteristics (inherited from the space-time contact elements bundle) with the natural contact structure of the isochrone contact elements bundle.

Proof. The space-time contact element at an isochrone's point is generated by the corresponding isochrone's contact element and by the direction of the projection of the characteristics line in the space-time. The isochrone's contact element velocity lies in the contact plane of the contact structure inherited from the space-time's contact structure, if and only if its contact point moves along the isochrone, being tangent to the contact element in the space-time. This occurs exactly when the contact point velocity belongs to a contact element of the isochrone. This means that both contact structures coincide.

Remark. The same reasoning works in a more general setting; we may substitute for the space-time isochrone any hypersurface in a manifold $M$, transversal to the projections of the characteristics of a fixed hypersurface in its contact elements bundle $P T^{*} M$ (for instance the result is still true in the pseudo-Riemannian case); we also may substitute for $P T^{*} M$ the bundle of transversally oriented contact elements (the spheres bundle, $S T^{*} M$ ).

DEFINITION. A Legendre manifold in a contact manifold is an integral manifold of the contact planes field having maximal dimension (that is dimension $n-1$ in a contact manifold of dimension $2 n-1$ ). 
Example. The set of all contact elements, tangent to a given submanifold (of arbitrary dimension) is a Legendre submanifold of the contact elements bundle of the ambient manifold.

For instance, fibres of the projective cotangent bundle are Legendre manifolds. The Legendre fibrations are fibrations of contact manifolds into Legendre fibres.

The front variety of a Legendre submanifold in a contact manifold with a Legendre fibration is its image under the projection to the base space of the fibration. Isotopies of a Legendre manifold generate motions and even metamorphoses of its front variety. These singularities of the fronts come from the projection. They are present even on the fronts travelling through smooth media. But if the medium contains an obstacle the Legendre submanifold itself may acquire singularities.

Example. Let us assume that the obstacle is bounded by a plane curve with a simple inflection point. The corresponding front (the curve evolvent) acquires a singularity of order $5 / 2$. Thus the corresponding variety of plane contact elements, i.e. the Legendre variety, consisting of all plane contact elements tangent to the front, is singular with a singularity of order $3 / 2$. In other words, the Legendre variety is locally diffeomorphic to a semicubical parabola in the 3-space.

\section{The contact triads}

The obstacle problem leads to the following definition motivated by the examples and theorems below.

Definition. A contact triad $(H, L, l)$ consists of

(1) a hypersurface $H$ in a contact manifold transversal to the contact planes;

(2) a Legendre manifold $L$ in the same contact manifold;

(3) a smooth hypersurface lin $L$, such that the hypersurface $H$ is tangent to the Legendre manifold $L$ with first order of tangency at every point of $l$.

We shall study the germ of a triad at a point 0 of $l$.

Definrtion. The Legendre variety, generated by the triad $(H, L, l)$ at 0 , is the image of the germ of $l$ at 0 by the projection of $H$ onto its space of characteristics.

Example 1. Let us extend the Euclidean space $R^{n}$ to the space-time $R^{n} \times R=\{q, t\}$. We call a space-time contact element non-vertical, if it may be defined by $d t=p d q$, and we call it a light contact element, if $p^{2}=1$. All light contact elements form a hypersurface $H$ in the bundle of contact elements over the space-time. This hypersurface $H$ is the first element in a triad defined by the family of geodesics on a hypersurface $\Gamma \subset R^{n}$, consisting of all geodesics, normal to a surface of codimension 1 in $\Gamma$.

Let $s: \Gamma \rightarrow R$ be the time function, defining the family of geodesics on $\Gamma$ (the geodesics are orthogonal to the surfaces $s=$ const, and $(\nabla s)^{2}=1$ on $\Gamma$ ). The graph of $s$ is a codimension-2 submanifold of space-time. Let us consider the set of all space-time contact elements, tangent to this graph. This set is a Legendre manifold, and it will serve as $L$ of the triad. 
Proposition 1. The hypersurface $H$ is (first order) tangent to the Legendre manifold $L$ along a submanifold l of codimension 1 in $L$, consisting of all contact elements, belonging to $L$, which contain the normal to $\Gamma \times t$ in $R^{n} \times t$.

Proof. The elements of $L$ at a point of the graph form a linear (one-parameter) family. Let us consider an element of $L$ defined by

$$
d t=p d x
$$

By the definition of $L$, the restriction of $p d x$ to $T_{x} \Gamma$ is $d s$. But

$$
(\nabla s)^{2}=1 \text {. }
$$

Hence the function $p^{2}$, restricted to the above-defined one-parameter family, has a non-degenerate minimum, equal to 1 ; it is attained for such a $p$, that

$$
p d x=0
$$

for all the vectors normal to $\Gamma$ in $R^{n}$.

Proposition 1 is proved: $(H, L, l)$ is a triad.

PROPOSITION 2. The Legendre variety generated by the triad constructed above, is the Legendre variety formed by those contact elements of $R^{n}$ which are tangent to the same front for the obstacle problem with boundary $\Gamma$.

Proof. The manifold of $H$-characteristics was identified in $\S 2$ with the bundle of transversally oriented contact elements of an isochrone $t=c$, that is with $S T^{*} R^{n}$. The characteristics intersecting $l$ are identified with those elements, normal to the tangent rays of geodesics of the family on $\Gamma$, which correspond to the isochrone $t=c$. An element corresponds to $t=c$, if and only if the oriented distance along the ray, from the point $x$ of contact of the ray with a geodesic to the contact point of the element, is equal to $c-s(x)$. These elements are precisely all elements tangent to the level variety $t=c$ of the (multi-valued) time function in $R^{n}$, defined by the family of geodesics on the obstacle surface. This proves proposition 2 . $\square$

We will now construct a series of triads examples, providing normal forms for the germs of generic triads at all their points. This implies, for instance, normal forms of singularities of the Legendre varieties, consisting of all contact elements tangent to a front for a generic obstacle in an Euclidean or Riemannian space.

\section{Contact structures on polynomials spaces}

Let $V=\left\{a_{0} x^{d}+\cdots+a_{d} y^{d}\right\}$ be the linear space of all real binary forms of degree $d$. The group SL $(2, R)$ of linear unimodular transformations of the $(x, y)$-plane acts on $V$. If $d$ is odd, the dimension of $V$, which is equal to $d+1$, is even and (as A. B. Guivental has remarked) $V$ carries a linear symplectic structure (a nondegenerate skew symmetrical bilinear form), invariant under the SL $(2, R)$ action; this form $\Omega$ is unique up to a non-zero multiple. The form $\Omega$ defines on $V$ a differential 2 -form $\omega$ with 'constant coefficients' which is a symplectic structure. 
This structure may be written as $\sum d p_{i} \wedge d q_{i}$ using suitable 'Darboux coordinates' in which the binary form $\varphi \in V$ with coordinates $\left(p_{i}, q_{i}\right)$ is defined by

$$
\varphi(x, 1)=q_{1} \frac{x^{d}}{d !}+\cdots+q_{n} \frac{x^{n}}{n !}-p_{n} \frac{x^{n-1}}{(n-1) !}+\cdots+(-1)^{n} p_{1},
$$

where $d=2 n-1$ and the signs before the $p_{k}$ 's alternate.

Let $v=\sum a_{i} \partial / \partial a_{i}$ be the Euler vector field on $V$. It is $\operatorname{SL}(2, R)$-invariant (every linear transformation sends $v$ to $v$ ). The interior product of $v$ with $\omega$ defines on $V$ an SL $(2, R)$-invariant differential 1 -form $i_{v} \omega$. This 1 -form is equal to zero on $v$. Hence it defines an $\operatorname{SL}(2, R)$-invariant hyperplane field in the projective space $R P^{d}$ of dimension 0 hypersurfaces of degree $d=2 n-1$. We obtain:

Proposition 1. The hyperplane field defined above is an $\operatorname{SL}(2, R)$-invariant contact structure on $R P^{d}$.

For instance, in the domain consisting of those polynomials $\varphi(x, 1)$ with $q_{1}=1$;

$$
\left\{f: f(x)=\frac{x^{2 n-1}}{(2 n-1) !}+q_{2} \frac{x^{2 n-2}}{(2 n-2) !}+\cdots+q_{n} \frac{x^{n}}{n !}-p_{n} \frac{x^{n-1}}{(n-1) !}+\cdots \pm p_{1}\right.
$$

the contact structure on the projective space is represented by the following 1 -form

$$
\alpha=p^{\prime} d q^{\prime}-q^{\prime} d p^{\prime}-d p_{1},
$$

where $q^{\prime}=\left(q_{2}, \ldots, q_{n}\right), p^{\prime}=\left(p_{2}, \ldots, p_{n}\right)$.

Let us consider a one-parameter group of contact diffeomorphisms. Points, where the velocity field vector lies in the contact plane, form a hypersurface given by the equation $\alpha \mid \xi=0$ if the velocity field is $\xi$ and the contact structure is $\alpha=0$. We will call this hypersurface the neutral surface of the group. Let us suppose, that the neutral hypersurface is transversal to the contact planes.

PROPOSITION 2. The orbits of the group on the neutral surface are its characteristics. Proof. By the homotopy formula,

$$
i_{\xi} d \alpha+d i_{\xi} \alpha=L_{\xi} \alpha
$$

The field $\xi$ being contact,

$$
L_{\xi} \alpha=0 \quad \text { if } \alpha=0
$$

On the neutral surface

$$
i_{\xi} \alpha=0
$$

Hence

$$
d i_{\xi} \alpha=0
$$

on its tangent vectors. Thus

$$
i_{\xi} d \alpha=0
$$

on the intersection of the contact plane with the tangent plane to the neutral surface, as required. 
The group of translations of polynomials along the $x$-axis acts on the space of polynomials of the form (*), and preserves its contact structure:

PROPOSITION 3. The neutral surface is defined by $K=0$, where

$$
K=p_{2}+p_{3} q_{2}+\cdots+p_{n} q_{n-1}+q_{n}^{2} / 2 .
$$

Indeed, the velocity field is

$$
\xi=\sum q_{k-1} \partial / \partial q_{k}-p_{k+1} \partial / \partial p_{k} \quad\left(p_{n+1}=q_{n}, q_{1}=1\right) .
$$

Proposition 4. The triple $H(K=0) ; L(p=0), l\left(p=q_{n}=0\right)$ is a contact triad.

This follows directly from the formula for $K$.

DFINITION. We call the standard variety of dimension $m$ and denote $\Sigma^{m}$ the Legendre variety generated by the triad of proposition 4 (here $m=n-2$ ).

COROLLARY. The standard Legendre variety $\Sigma^{n-2}$ consists of those polynomials of the form:

$$
\frac{x^{2 n-1}}{(2 n-1) !}+q_{3} \frac{x^{2 n-3}}{(2 n-3) !}+\cdots+q_{n} \frac{x^{n}}{n !}-p_{n} \frac{x^{n-1}}{(n-1) !}+\cdots+(-1)^{n} p_{1}
$$

where $p_{2}$ means $-\left(p_{4} q_{3}+\cdots+p_{n} q_{n-1}+q_{n}^{2} / 2\right)$, which have a root of multiplicity greater than $n$; the contact structure is defined by the 1-form

$$
\alpha=p^{\prime \prime} d q^{\prime \prime}-q^{\prime \prime} d p^{\prime \prime}-d p_{1},
$$

where $q^{\prime \prime}=\left(q_{3}, \ldots, q_{n}\right), p^{\prime \prime}=\left(p_{3}, \ldots, p_{n}\right)$.

From this corollary and from [5] follows:

COROLlaRY. The standard Legendre variety $\Sigma^{m}$ is diffeomorphic to the open swallowtail of dimension $m$, that is to the variety of all polynomials:

$$
x^{2 m+1}+A_{1} x^{2 m-1}+\cdots+A_{2 m}
$$

which have a root of multiplicity at least $m+1$.

Example. Let $n=3$. Formula (**) implies

$$
\begin{gathered}
f=\frac{x^{5}}{120}+q_{3} \frac{x^{3}}{6}-p_{3} \frac{x^{2}}{2}-\frac{q_{3}^{2}}{2} x-p_{1}, \\
\alpha=p_{3} d q_{3}-q_{3} d p_{3}-d p_{1} .
\end{gathered}
$$

The polynomials which have roots of multiplicity greater than 3 form a Legendre curve

$$
\Sigma^{1}=\left\{f=(x-t)^{4}(x+4 t) / 120\right\}
$$

that is

$$
q_{3}=-t^{2} / 2, \quad p_{3}=-t^{3} / 3, \quad p_{1}=-t^{5} / 30
$$

This curve is diffeomorphic to a cubic parabola. Its singularity is responsible for the singulariites of order $5 / 2$ of evolvents at the inflections tangent. 
Remark. One sees from (**), that the variety $\Sigma^{n-2}$ is quasi-homogeneous, for the coordinates weights

$$
\begin{gathered}
\operatorname{deg} q_{i}=i-1, \quad \operatorname{deg} p_{i}=2 n-i \\
(\operatorname{deg} K=2 n-2, \quad \operatorname{deg} \alpha=2 n-1) .
\end{gathered}
$$

The standard variety $\Sigma^{n-2}$ is the image of a space with coordinates of weights $(1, \ldots, n-2)$ under a quasi-homogeneous mapping.

Example. For $n=4$ the coordinates $\left(q_{3}, q_{4}, p_{4}, p_{3}, p_{1}\right)$ have weights $(2,3,4,5,7)$. The Legendre variety $\Sigma^{2}$ is the image of a plane $(t, r)$, where $\operatorname{deg} t=1, \operatorname{deg} r=2$ :

$$
\Sigma^{2}=\left\{f=\left(x^{2}+5 t x+r\right)(x-t)^{5} / 7 !\right\} \text {. }
$$

\section{Contact triads normal forms}

Let us consider the space $R^{2 k+1}$ equipped with the contact structure $\alpha=0$,

$$
\alpha=p^{\prime} d q^{\prime}-q^{\prime} d p^{\prime}-d p_{1},
$$

where $p^{\prime}=\left(p_{2}, \ldots, p_{k}\right) ; q^{\prime}=\left(q_{2}, \ldots q_{k}\right)$.

DEFINITION. The standard contact triad $\tau_{k, n}(n \leq k)$ is the triad $(H, L, l)$ in this space, defined by the equations

$$
\begin{gathered}
H: \quad p_{2}+p_{3} q_{2}+\cdots+p_{n} q_{n-1}+q_{n}^{2} / 2=0 \\
L: \quad p=0 ; \quad l: \quad p=0, q_{n}=0 .
\end{gathered}
$$

Remark. The triad $\tau_{k, n}$ is a trivial extension (by addition of new $p$ 's and $q$ 's) of $\tau_{n, n}$, the triad studied in $\$ 4$.

THEOREM 1. The germs at 0 of the standard triads $\tau_{k, n}$ are stable, as germs of contact triads considered up to the contact equivalence.

THEOREM 2. The germs of generic contact triad at all points are contact equivalent to the germs of standard triads at 0.

COROLLARY. The germs of the Legendre variety, formed by the contact elements tangent to a front in the generic obstacle problem (see $\$ \$ 3$ and 4) are contact stable and contact equivalent to the germs of standard Legendre varieties (may be multiplied by the linear spaces) - and hence are diffeomorphic to the germs of open swallowtails.

Proof. We need a simple lemma:

LEMMA 1. In a neighbourhood of a point, where a hypersurface in a contact space is transversal to the contact plane, one may choose an equation $\alpha=0$ for the contact planes in such a way, that the kernel of the 2-form do is tangent to the hypersurface.

Proof. One may reduce the equation of the hypersurface germ to the form $P_{2}=0$, the contact structure being $P^{\prime} d Q^{\prime}-d P_{1}$. This follows, for instance, from the Guivental-Darboux theorem [2], which says that in contact geometry there is no extrinsic curvatures: the traces of the contact planes on the germ of a submanifold define the germ up to a contact equivalence, and define the restriction of a contact form up to a contact form preserving equivalence. 
We apply the lemma to the $H$ of a contact triad. The field of kernels of $d \alpha$ defines (locally) a fibration of the contact space into curves (in terms of the above coordinates: $\left.\left(P^{\prime}, Q^{\prime}, P_{1}\right) \mapsto\left(P^{\prime}, Q^{\prime}\right)\right)$. The base $B$ of this fibration $\pi$ has a natural symplectic structure defined by the projection of the 2 -form $d \alpha$ along its kernels' integral curves. The hypersurface $H$ is fibred into these integral curves. Hence this hypersurface is projected onto a hypersurface $\pi H$ in $B$.

The kernel of the 2 -form $d \alpha$ is not tangent to the Legendre manifold $L$, because its tangent plane belongs to the contact plane $\alpha=0$, which is transversal to the kernel of $d \alpha$ by the definition of a contact structure.

Hence the projection of the triad $(H, L, l)$ to the symplectic manifold $B$ defined above is the triple $(\pi H, \pi L, \pi l)$, where $H$ is a hypersurface in $B$, first order tangent to the submanifold $\pi L$ along its hypersurface $\pi l$. Note, that germs of $L$ and of $l$ are projected diffeomorphically.

$\pi L$ is a Lagrange manifold because $L$ is a Legendre manifold. Thus the triple is a symplectic triad in $B$ and the symplectic case of our theorem is known (A. B. Guivental). Thus we obtain normal forms of the projections of our triads to the $\left(P^{\prime}, Q^{\prime}\right)$ space.

The normal form for a contact triad follows because $P_{1}=\int P^{\prime} d Q^{\prime}$ (integrals along paths on the Lagrange manifold $\pi L$, starting at 0 ). So we have derived the classification of the contact triads from that of symplectic triads.

\section{REFERENCES}

[1] O. A. Platonova. Singularities in the shortest way problem at an obstacle. Funct. Anal. 15 (1981), 86-87.

[2] V. I Arnol'd. Catastrophes theory. Znanie: Moscow, 1981. (In Russian.)

[3] V. I. Arnol'd. Evolution of singularities of potential flows in collision free media and caustics metamorphosae in 3-space. Trudy semin. im. I. G. Petrovskogo 8 (1982), 21-57. (In Russian.)

[4] V. I. Arnol'd. Lagrange varieties, asymptotical rays and the open swallowtail. Funct. Anal. 15 (1981), 1-14.

[5] A. B. Guivental. Varieties of polynomials having a prescribed multiplicity root and the generalized Newton equation. Funct. Anal. 16 (1982), 8-13.

[6] V. I. Arnol'd. Mathematical Methods of Classical Mechanics. Springer: New York, 1978.

[7] V. I. Arnol'd, A. N. Varchenko \& S. M. Gusein-Zade. Singularities of Differentiable Mappings. Nauka: Moscow, 1982. (In Russian.) 